\title{
Role of Body Area Sensor Networks in Smart Health Care
}

\author{
Neelanjana Basu Roy and Debasis Das
}

\begin{abstract}
The term "computer" or "PC" has never yielded another term "personal". It is just regarded as a machine with inputs from human beings giving required outputs. However the term or the budding field of Body Area Networks gives new meaning or leverage now to the term "personal" in PCs. In short, this wireless technology leverages wireless communications protocols allowing low-powered sensors to communicate with one another and transmit data to a local base station and to remote places like hospitals. This paper on Wireless Body Area Networks will present a detailed discussion on the various applications of WBANs in smart healthcare systems, its history of development, advantages, disadvantages and the future prospect and scenario of this system. We will also highlight project performed earlier that helped WBANs to provide long-term healthcare monitoring. Our main aim in this paper, is to understand one of the major applications of Body Area Sensor Networks, termed as "Amplifier Ear" which helps people suffering from auditory problems to function properly.
\end{abstract}

Index Terms-Amplifier ear, body area network (BAN), body sensor networks (BSNs), wireless sensor networks (WSNs).

\section{INTRODUCTION}

With the advancement of technology, human beings have always tried to reduce the size of digital communication to such an extent that it can be portable as well as easy to use. Thus, devices like cellular phones, pagers and MP3 players have become popular; people started to carry such devices around their bodies. With such an advent, studies ensued whether electronic devices could be operated on and near human body. It was during this time that the idea of Body Area Network or BAN was invented.

A formal definition of Body Area Networks by IEEE 802.15 is "a communication standard optimized for low power devices and operation on, in or around the human body (but not limited to humans) to serve a variety of applications including medical, consumer electronics/personal entertainment and other" BAN devices can be of the following types

1) May be embedded inside the body, in form of implants,

2) May be surface-mounted on the body in a fixed position, also known as Wearable technology,

3) May be accompanied devices which humans can carry in different positions, in clothes, by hand or in various bags.

4) Used in hospitals to allow monitoring technology and collect patient data BAN is used in healthcare network for continuously monitoring the patient

Manuscript received March 16, 2015; revised September 21, 2015. This work was supported by a fund (NFS2015DTB01) from the forensic research program of the National Forensic Service, Korea.

The authors are with NIIT University, India (e-mail: eelanjanab.roy@st.niituniversity.in). where various sensors are attached to the patient's body. Values are taken by various sensors and then analysed. It is very easy for patient to make physical movement (see Fig. 1).

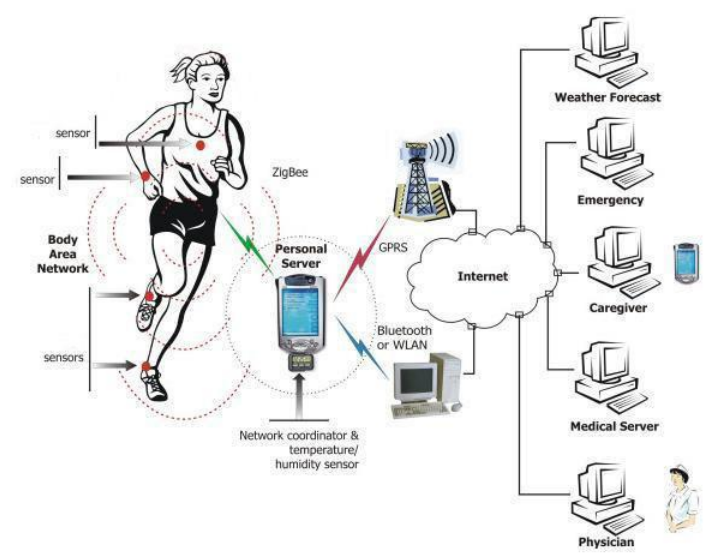

Fig. 1. Various uses of body area network for smart healthcare.

\section{PRELIMINARIES}

Since BAN is still developing, it has very little amount of history attached to it. It has emerged from the basic ideas of sensor network and biomedical engineering. The term body sensor network was formally coined for the first time in 2006 by professor Guang-Zhong yang in his book [1]. This technology represents the lower bound of power and bandwidth from the ban use case scenarios. BAN technology is quite flexible and there are many potential uses for ban technology in addition to BSNs. Some of the more common use cases for ban technology are [1]: personal devices, body sensor networks (bsn), wireless audio, sports and fitness monitoring and mobile device integration.

Each of these use cases have unique requirements in terms of bandwidth, latency, power usage, and signal distance. Given below are the data rates vs. power, according to IEEE-BAN Summary (see Fig. 2).

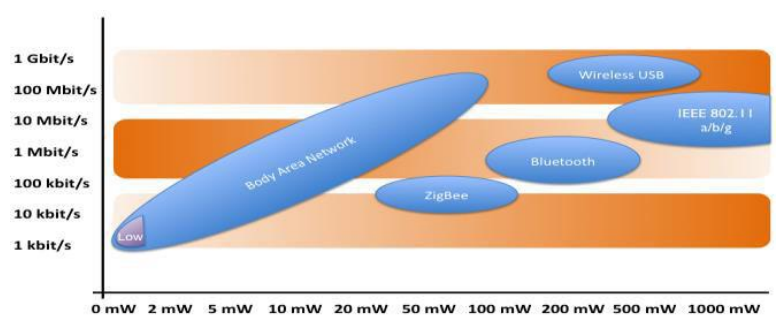

Fig. 2. A graph explain date rate vs, power in WBAN.

The types of devices used in the Body AreaNetwork are as follows [1]:

1) Wireless Sensor Node: A network thatgathers information based on physical stimuli, it allows gathering and process of information really fast. the sensors or from the user. 
2) Wireless Actuator Node: A device acting according to data received by the sensors or from the user.

3) Wireless Personal Device: A device that gathers information using the above sensors and actuators and informs the patient about his/her current health condition.

\section{RELATED/EXISTING WORK}

We shall now talk about the applications of Body Area Network that have found success in the field of Smart HealthCare.

\section{A. Heart-Monitoring System}

We can install smart sensor nodes on the patient in an inconspicuous way can prevent a large number of deaths caused by cardiovascular diseases every year [2]. The medical staff can do treatment preparation in advance as soon as they receive vital information regarding heart rate and irregularities of the heart while monitoring the health status of the patient, and there is no delay if the patient is suddenly in a critical condition.

\section{B. Cancer Detection}

One of the leading causes of death in the world today is associated with rising numbers of cancer patients each year and nowadays one of the biggest threats for human life is cancer [3]. A sensor with the ability to detect nitric oxide (which is emitted by cancer cells) can be placed by doctors where they identify as the suspect locations. These sensors have the ability to differentiate cancerous cells, between different types of cells, thus allowing medical staff to detect cancer at a very early stage.

\section{Hip-Guard System}

Hip-Guard system [3] is a system basically developed for those patients who are recovering from hip surgery. This system monitors patient's leg and hip position and rotation with the help of embedded wireless sensors. Alarm signals can be sent to patient's Wrist Unit if hip or leg positions or rotations turn out to be false, and hence Hip-Guard system provide useful real-time information for patient recovery process.

\section{Mobihealth}

Mobihealth [3] is a project using GPRS/UMTS wireless communication technology [4] that is used for transferring data, to create a generic platform for home healthcare using BAN-Based sensors and wireless telephony technology. This aims to provide continuous monitoring to patients outside the hospital environment. MobiHealth [4] targets, improving the quality of life of patients by enabling new value added services in the areas of disease prevention, disease diagnosis, and remote assistance, physical state monitoring and even in clinical research. Therefore, a patient who requires monitoring for short or long periods of time doesn't have to stay in hospital for monitoring. With the MobiHealth BAN the patient can be free to pursue daily life activities. However, though this project is still undergoing research, it is expected soon to be used around the world, especially at remote places where medical support is difficult to find (see Fig. 3).

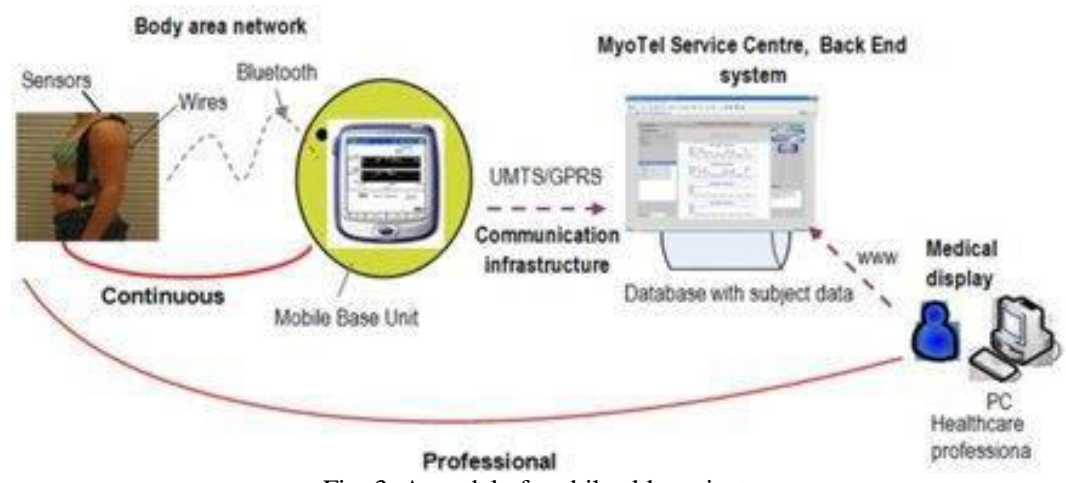

Fig. 3. A model of mobihealth project.
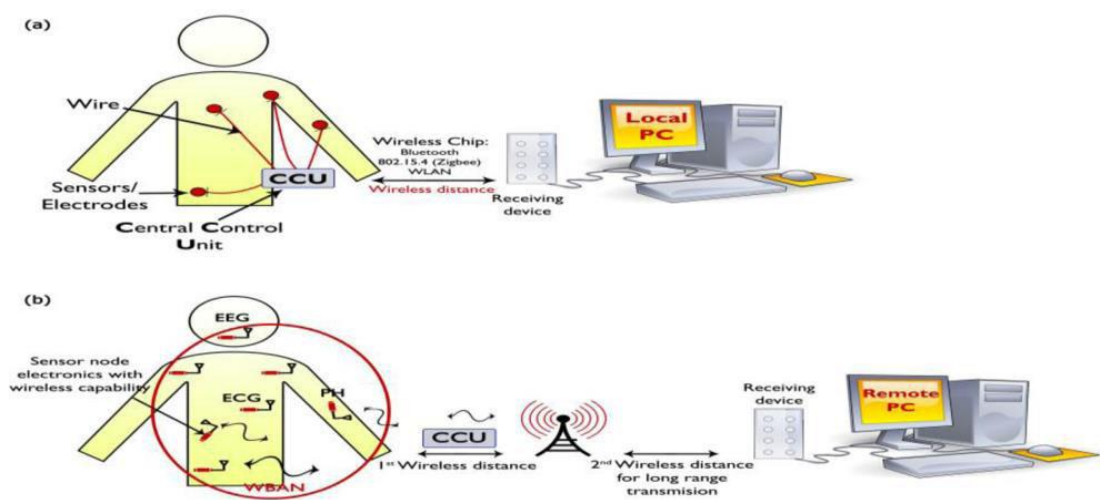

Fig. 4. A model of LifeShirt.

\section{E. Asthma}

A wireless sensor network [4] can help those patients suffering from asthma by having sensor nodes that can sense the allergic agents in the air and report the status continuously to the physician and/or to the patient himself, to take necessary precaution (see Fig. 4). 


\section{F. LifeShirt}

LifeShirt [4] is a comfortable and completely non-invasive "smart garment" that gathers data during a patient's daily routine, providing the most complete remote picture of a patient's health status. It enables healthcare professionals and researchers to accurately monitor more than 30 vital life-sign functions in the real-world settings where patients live and work. LifeShirt collects patient data using integrated sensors including respiratory bands (which measure pulmonary function) and an ECG (which record electrical activity of the heart). It also tracks and records posture and physical activity.

\section{G. Glucose Level Monitoring}

In the current world scenario, Diabetes has evolved to be one of the primary diseases, especially to office- goers. Diabetes can cause other complicated diseases like heart disease, stroke, high blood pressure, blindness, kidney disease, and last but not least amputations. Thus, how could we avoid this scenario? A recent study says that use of biosensor implanted within patient body could lead to better health solutions and provide a more consistent, accurate, and less invasive method by monitoring glucose levels, transmit the results to a wireless Personal Digital Assistant or a fixed terminal, and by injecting insulin automatically when a threshold glucose level is reached. All these could be achieved even when the doctor is not around.

\section{H. Prosthetic Arm}

A prosthesis is an artificial device helping to replace a body part, which may be lost through trauma, disease, or congenital conditions and has wireless networks incorporated in it. For example, a transradial prosthesis is an artificial limb that replaces an arm missing below the elbow. One type of prosthetics available are myoelectric arms which work by sensing, via electrodes. When the muscles in the upper arm moves, it causes an artificial hand to openor close. In the prosthetic industry a trans-radial prosthetic arm is often referred to as a"BE" or below elbow prosthesis.

\section{ADVANTAGES AND DisADVANTAGES}

Wired vs. Wireless: The most frequently used option these days is wireless connection like Bluetooth or Zigbee. But, Wireless Network have certain disadvantages like power challenge, computation, material constraints, robustness, security and interference, non-existent support for massive data, interferences of multidevices sharing the channel and lack of integration sensors.

\section{PROPOSED MODEL}

Often we find people with hearing disability not able to participate in conversations properly, or not communicate effectively at work, and annoy others who have to repeat themselves. To reduce this we present an effective method using wireless sensor networking in body area networks [5], [6] the "Amplifier Ear". This amplifier ear circuit, connected to $32 \mathrm{Ohm}$ impedance mini-earphones, can detect very remote sounds. Useful for theatre, cinema and lecture goers: every word will be clearly heard. You can also listen to your television set at a very low volume, avoiding to bother relatives and neighbors. Even if you have a faultless hearing, you may discover unexpected sounds using this device: a remote bird twittering will seem very close to you (see Fig. 5).

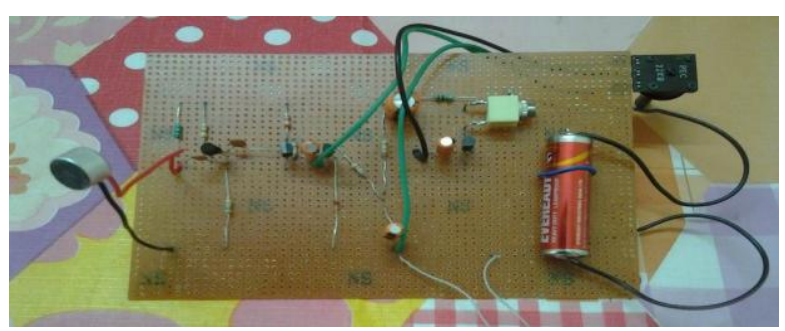

Fig. 5. Live model of amplifier ear.

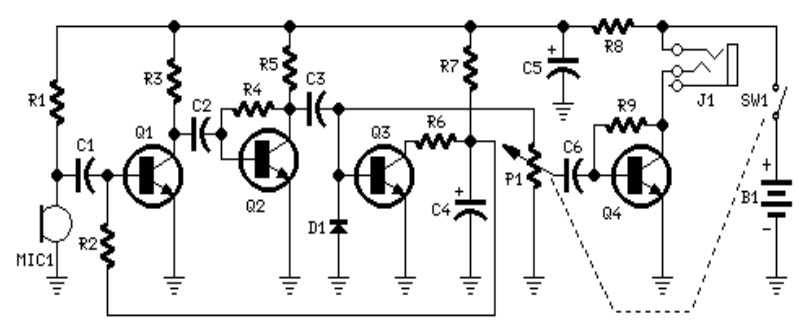

Fig. 6. Circuit model of amplifier ear.

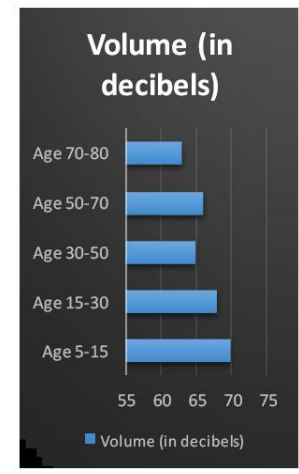

Fig. 7. Simulation result chart.

\section{GenetiC AlgORITHM}

The algorithm is as follows:

Step 1: Attach the capacitors and resistors as shown in Fig. 6.

Step 2: The heart of the circuit is a constant-volume control amplifier. All the signals picked-up by the microphone are amplified at a constant level of about 1 Volt peak to peak.

Step 3: Very low amplitude audio signals are highly amplified and high amplitude ones are limited. This operation is accomplished by Q3, modifying the bias of Q1 (hence its AC gain) by means of R2.

Step 4: Repeat this procedure till you feel you have the perfect decibel.

Step 5: To stop the function, just unplug the headphones from the jack.

\section{SimUlation AND RESUlT}

It has been observed and reported that the Amplified Ear is so sensitive that it can hear a whisper 7 meters across the room. If we hook a small relay coil to the input and able to locate power lines in the wall, we will be able to hear the neighbor's stereo perfectly. It is suggested that this circuit could make 
also a good electronic stethoscope. It could also make a very good wireless auditory device for people having problem in sensory reception of the ear (see Fig. 7).

\section{CONCLUSIONS}

The biggest benefit to having a wireless network is that it allows providers to deploy technology at the bedside, as part of normal health care workflow. Also used for detection of chronic diseases beforehand [7]. It can be used in military for security purposes assists seamless communication between individual and machine. This paper has thus, helped review the basic research on Wireless Body Area Networks in Smart Health Care. This work provides an overview on research propagation within human body. As explained above, a WBAN is expected to have great beneficial results on patients' bodies, helping them track any unusual effect on the body and allow early detection of problems. With the technological evolution it is expected very soon, the medical aspect of the world will take new turn with physical large monitoring devices being replaced by wireless networking system and WBAN will play an important role in the long run.

\section{REFERENCES}

[1] E. Karulf, "Body area networks (BAN)," IEEE 802.15, pp. 2-3, 2008

[2] M. C. Navale and R. T. Chavan, "A survey paper on body area network in healthcare system," Multidisciplinary Journal of Research in Engineering and Technology, vol. 1, issue 2, pp. 149-150, 2014.

[3] M. P. Khan, A. Hussain, and K. S. Kwak, "Medical applications of wireless body area networks," International Journal of Digital Content Technology and Its Applications, vol. 3, no. 3, pp. 185-189, September 2009
[4] D. M. M. P. Schreurs, M. Mercuri, P. J. Soh, and G. A. E Vandenbosch, "Wireless health monitoring: Design challenges,", 2013 11th International Conference on Telecommunication in Modern Satellite, Cable and Broadcasting Services (TELSIKS), vol. 2, pp 351-359, Oct. 16-19, 2013.

[5] G. F. Fang, E. Dutkiewicz, M. A. Huq, R. Vesilo, and Y. H. Yang, "Medical Body Area Networks: Opportunities, challenges and practices," Communications and Information Technologies (ISCIT), in Proc. 11th International Symposium, 2011, pp. 562-567.

[6] P. V. Torre, P. Vanveerdeghem, H. Rogier, and I. Moerman, "Energy-efficient off-body communication nodes with receive diversity," in Proc. 2013 IEEE 20 ${ }^{\text {th }}$ Symposium on Communications and Vehicular Technology in the Benelux (SCVT), 2013, pp. 1-6.

[7] D. Das and R. Misra, "Approximating Geographic routing using coverage tree heuristics for wireless network," Wireless Networks (WINE), Springer, 2014.

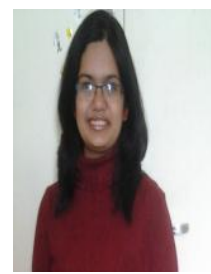

Neelanjana Basu Roy is currently pursuing her B.Tech. degree (3rd Year) in Computer Science and Engineering Department in NIIT University. Her research interests include computer network, algorithm, network security, wireless networking. She has published a research paper in International Conference on Electrical, Electronics, Signals, Communication and Optimization (EESCO) under IEEE in Vishakhapatnam, India in January, 2015.

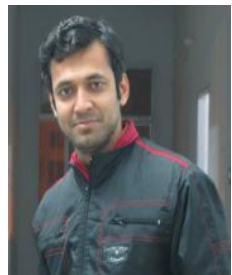

Debasis Das is an assistant professor in Computer Science and Engineering Department in NIIT University, Neemrana, Rajasthan, India. $\mathrm{He}$ is pursuing the Ph.D in vehicular ad-hoc networks (VANETs) from the Department of Computer Science and Engineering, Indian Institute of Technology (IIT) Patna, Bihar, India. He received his M.Tech in computer science and engineering degree from School of Computer Engineering, KIIT University, Bhubaneswar, India. His research interests include computer networks, vehicular network, algorithm, network security and cellular automata. 